\title{
THE UNIT GROUPS OF AFFINE ALGEBRAIC MONOIDS ${ }^{1}$
}

\author{
WILLIAM C. WATERHOUSE
}

\begin{abstract}
An affine algebraic group can be embedded as a proper dense subgroup of an affine algebraic monoid iff it has a homomorphism onto the multiplicative group.
\end{abstract}

Consider affine algebraic monoids over an algebraically closed field. Such monoids have been studied in a series of papers by M. S. Putcha $[3,4,5,6,7,8]$, who has shown (among other things) that the idempotents and other features of the monoid may strikingly reflect nontrivial properties of the group of units. But he has not analyzed what restrictions there are on these unit groups-or, to put in another way, what groups might be studied by embedding them in monoids. That question is answered here.

THEOREM. Let $G$ be a connected affine algebraic group over an algebraically closed field. The following are equivalent:

(1) $G$ is the group of units of an affine algebraic monoid that is a connected set and is not equal to $G$.

(2) $G$ is the group of units of a nontrivial affine algebraic monoid that is an irreducible set and has a zero.

(3) $G$ has a nontrivial character (homomorphism to $G_{m}$ ).

Proof. Clearly (2) implies (1). Assume (1), and let $S$ be the monoid. By [1, II, 2, 3.3 , p. 183] we may embed $S$ as a closed submonoid of some matrix monoid $M_{n}(k)$. [This holds for monoids by the same proof as for groups: a finite set of algebra generators for $k[S]$ will be contained in a finite-dimensional subcomodule, which gives a faithful linear representation.] Then $S \cap \mathrm{GL}_{n}(k)$ is a closed submonoid of $\mathrm{GL}_{n}(k)$, and hence it is a subgroup [1, II, 2, 3.5], so $G=S \cap \mathrm{GL}_{n}(k)$, and $G$ is open in $S$. The determinant here gives a homomorphism $G \rightarrow G_{m}$. If it is trivial on $G$, then $G=S \cap \mathrm{SL}_{n}(k)$, and $G$ is closed in $S$. Since $S$ is connected and not equal to $G$, this is impossible. Hence $G$ has a nontrivial character.

Assume now (3), and let $\varphi: G \rightarrow G_{m}$ be a nontrivial character. As $G$ is connected, $\varphi(G)=G_{m}$, and hence by [2, p. 136] there is a maximal torus $T \cong G_{m}^{s}$ of $G$ mapping onto $G_{m}$. Therefore there is a closed subgroup $H$ of $G$ isomorphic to $G_{m}$ on which $\varphi$

Received by the editors May 14, 1981 and, in revised form, October 14, 1981.

1980 Mathematics Subject Classification. Primary 20G15, 20M10.

'This work was supported in part by the National Science Foundation. 
is nontrivial. If $\psi: G_{m} \stackrel{\sim}{\rightarrow} H$ is an isomorphism, then $\varphi \psi(a)=a^{r}$ for some $r \neq 0$; replacing $\psi$ by its reciprocal if necessary, we may assume $r>0$.

Embed $G$ as a closed subgroup of some $\mathrm{GL}_{n}(k)$. As $H$ is a diagonalizable group, we can choose coordinates so as to have a basis $\left\{e_{i}\right\}$ of $k^{n}$ for which $\psi(a) e_{i}=a^{m(i)} e_{i}$ for various integers $m(i)$. They may not all be positive, and to remedy that we look at the action of $G$ on $k^{n} \oplus k$ given by

$$
g \cdot(x, \lambda)=\left(\varphi(g)^{N} g(x), \varphi(g)^{N} \lambda\right)
$$

for some large positive integer $N$. It is easy to check that this is indeed a representation of $G$. If $g$ acts trivially, then from the second component we see $\varphi(g)^{N}=1$; from the first component then we see $g(x)=x$ for all $x$, so $g$ is trivial. Thus the representation is faithful, and hence it embeds $G$ as a closed subgroup of $\mathrm{GL}_{n+1}(k)$.

An element $\psi(a)$ of $H$ in this embedding becomes the diagonal matrix

$$
\operatorname{diag}\left\langle a^{m(1)+N r}, \ldots, a^{m(n)+N r}, a^{N r}\right\rangle \text {. }
$$

As $N$ is large and $r$ is positive, all the exponents here are strictly positive. Hence the map extends to $a=0$, and the matrix identically zero in $M_{n+1}(k)$ is in the closure of $H$. Take then the closure $S$ of $G$ in $M_{n+1}(k)$. It is automatically an algebraic monoid with $G$ as group of units; it is irreducible, since $G$ is; and it contains a zero, the zero of $M_{n+1}(k)$.

Corollary (Putcha [7, TheORem 3]). If G satisfies condition (2) of the theorem and its maximal torus has dimension 1, then $G$ is solvable.

Proof. The condition on the torus implies that $G$ modulo its unipotent radical must be $G_{m}$ or $S L_{2}$. In the first case it is solvable, and in the second case it has no characters.

REMARKS ON GENERALIZATIONS. (1) In the theorem we have followed Putcha in assuming that the base field is algebraically closed, but actually the result is true for algebraic matrix groups over any infinite field $k$. The only step in the argument that needs to be modified is the.proof that there is an $H \simeq G_{m}$ on which $\varphi$ is nontrivial. For this we recall [2, p. 220] that the algebraic group has a maximal torus $T$ defined over $k$. All other maximal tori are conjugate to $T$ over $\bar{k}$, so $T$ cannot be in the kernel of $\varphi$. Up to isogeny $T$ is a product of split and anisotropic subtori, and the split torus factor then contains a copy of $G_{m}$ on which $\varphi$ is nontrivial.

(2) Carrying over the language familiar for groups, Putcha calls an affine algebraic monoid "connected" only when as a space it is actually irreducible. For monoids, unlike groups, this is an abus de langage: the closed set $\{(x, y) \mid x= \pm y\}$ in $k^{2}$, for instance, is a monoid under coordinatewise multiplication and (for $\operatorname{char}(k) \neq 2)$ is connected but reducible. The advantage of Putcha's terminology is that irreducibility forces the group of units to be connected, while (as the above example shows) connectedness of the monoid does not. In our theorem we got the same groups either way because we assumed $G$ connected to begin with. If we drop that assumption, we get the following result. 
THEOREM. Let $G$ be an affine algebraic group over an algebraically closed field. The following are equivalent:

(1) $G$ is a dense subgroup of an affine algebraic monoid that is not equal to $G$.

(2) $G$ is the group of units of a nontrivial affine algebraic monoid that is a connected set and has a zero.

(3) $G$ has a character of infinite order (homomorphism onto $G_{m}$ ).

The argument for this is quite parallel to that for our original theorem. For (1) $\Rightarrow(3)$ we observe that the matrices with determinants in a finite subgroup of $G_{m}$ are a closed set in $M_{n}(k)$, and hence det must be of infinite order on $G$. For (3) $\Rightarrow(2)$ we note that any $g$ in $G$ lies in the connected set $g H$, which has $g \cdot 0=0$ in its closure in $M_{n+1}(k)$; thus all elements of $G$ are connected to zero, and the closure of $G$ is connected.

Note that this theorem may rule out $G$ even when $G^{\circ}$ can occur; if for instance $G$ is the semidirect product of $G_{m}$ and $\langle h\rangle$ with $h$ of order two acting on $G_{m}$ by inversion, then $G$ modulo its commutator subgroup is finite.

\section{REFERENCES}

1. M. Demazure and P. Gabriel, Groupes algébriques. I, North-Holland, Amsterdam, 1970.

2. J. Humphreys, Linear algebraic groups, Springer-Verlag, New York, 1975.

3. M. S. Putcha, On linear algebraic semigroups, Trans. Amer. Math. Soc. 259 (1980), 457-469.

4. __ On linear algebraic semigroups. II, Trans. Amer. Math. Soc. 259 (1980), 471-491.

5. __, On linear algebraic semigroups. III, Internat. J. Math. Math. Sci. 4 (1981), 667-690.

6. __ Green's relations on a connected algebraic monoid (to appear).

7. The group of units of a connected algebraic monoid (to appear).

8. Connected algebraic monoids (to appear).

Department of Mathematics, Pennsylvania State University, University Park, Pennsylvania 16802 